\title{
Collective Modes in a Bilayer Dipolar Fermi Gas and the Dissipationless Drag Effect
}

\author{
B. Tanatar
}

Received: 7 July 2012 / Accepted: 26 October 2012 / Published online: 9 November 2012

(C) Springer Science+Business Media New York 2012

\begin{abstract}
We consider the collective modes of a bilayer dipolar Fermi system in which the particles interact via long range $\left(\sim 1 / r^{3}\right)$ interaction. Assuming that each layer has a background flow which varies little and that the dynamics of the superfluid near $T=0$ is the same as that of a normal fluid, we obtain the dispersion relations for the collective modes in the presence of background flow. Decomposing the background flow into two parts, the center-of-mass flow and counterflow, we focus on the properties of the counterflow. We first find an estimate of the change in the zero-point energy $\Delta E_{\mathrm{ZP}}$ due to counterflow for a unit area of bilayer. Combining this with the free energy $F$ of the system and taking the partial derivatives with respect to background velocities in the layers, we determine the current densities which reveal the fact that current in one layer does not only depend on the velocity in the same layer but also on the velocity of the other layer. This is the drag effect and we calculate the drag coefficient.
\end{abstract}

Keywords Bilayer dipolar Fermi gas · Collective modes · Drag effect

\section{Introduction}

Ultracold gases of polar atoms or molecules with their anisotropic long-range interaction are attracting a lot of interest in recent years [1, 2]. In this context, single and multi-layer configurations of two-dimensional bosons or fermions are being studied from the point of view of their quantum phases, Fermi liquid properties, collective excitations, and formation of density waves [3-11].

In electronic bilayer systems a transport phenomenon known as the drag effect has been studied for a long time. In the drag effect when a current is applied to one of

\footnotetext{
B. Tanatar $(\bowtie)$

Department of Physics, Bilkent University, Bilkent, 06800, Ankara, Turkey

e-mail: tanatar@fen.bilkent.edu.tr
} 
the layers the electrons in the second will be dragged resulting in an interlayer resistivity which is related to the momentum transfer between the layers $[12,13]$. On the other hand, Duan in a series of papers [14-16] introduced the idea of dissipationless drag at $T=0$ between two superconducting layers. This has been later extended to bilayer charged and neutral boson systems [17-19]. The superflow/supercurrent drag effect has in fact been discussed earlier for a superfluid ${ }^{3} \mathrm{He}-{ }^{4} \mathrm{He}$ mixture [20] and proton-neutron mixtures in neutron stars [21]. On the experimental side, supercurrent drag effect between a normal metal or a semiconductor and a superconducting film separated by an insulating layer has been observed [22-24].

In this paper we study the supercurrent/superflow drag effect in bilayer dipolar Fermi systems. We consider two identical, superfluid layers of dipolar fermions separated by a distance $a$ at $T=0$. Calculating the zero-point energy with the help of the collective modes with and without the counterflow velocity to leading order, we find that the current in one layer not only depends on the velocity in the same layer but also on the velocity in the other layer. This is the hallmark of the drag effect. It is interesting to note that there has been recent considerations of counterflow effects in superfluid Bose systems [25] and of frictional drag effect in trapped dipolar gases [26].

The rest of this paper is organized as follows. We describe the model in Sect. 2 and present our collective mode and drag analyses for bilayer dipolar fermions in Sect. 3 . We end with brief concluding remarks in Sect. 4.

\section{Model}

We consider a bilayer system of dipoles with uniform density $n$ in each layer. The interaction for dipoles in the same layer is given by

$$
V_{11}(r)=\frac{C_{d d}}{4 \pi} \frac{1}{r^{3}}
$$

where $C_{d d}$ is the dipole-dipole coupling whose origin depends either on the electric dipole moment or the magnetic dipole moment of the particles. For simplicity we assumed that dipole moments are all oriented in the $z$-direction, thus the interaction is isotropic. We have also neglected the finite thickness of the layers in which case the intra-layer interaction in Fourier space reads [9, 10]

$$
V_{11}(q)=V_{0}-\frac{C_{d d}}{2} q
$$

where $V_{0}$ is a short-range interaction originating from the short distance cut-off in the Fourier transform. As the physical properties should not depend on this cut-off we regard $V_{11}(q)$ as an effective interaction. We, thus, introduce correlations described by the Hubbard approximation ${ }^{1}$ which has the form $G_{H}(q)=V_{11}\left(\sqrt{q^{2}+k_{F}^{2}}\right) / V(q)$

\footnotetext{
${ }^{1}$ This approximation is often used in the context of correlations in electron liquids, see for instance [27].
} 
and our effective interaction becomes $V_{11}(q) \rightarrow V_{11}(q)\left[1-G_{H}(q)\right]$, or more explicitly

$$
V_{11}(q)=\frac{C_{d d}}{2}\left[\sqrt{q^{2}+k_{F}^{2}}-q\right]
$$

which is now regular.

The inter-layer interaction, on the other hand, is given by

$$
V_{12}(r)=d^{2} \frac{r^{2}-2 a^{2}}{\left(r^{2}+a^{2}\right)^{5 / 2}}
$$

where $a$ is the separation distance between the layers, whose Fourier transform is

$$
V_{12}(q)=-\frac{C_{d d}}{2} q e^{-a q}
$$

There is no need to introduce an effective interaction for $V_{12}(q)$ as it is already regular at this level of approximation. Note that the inter-layer interaction is attractive, making the situation similar to bilayer electron-hole system. We introduce a length scale $r_{0}=C_{d d} m /\left(4 \pi \hbar^{2}\right)$ and use the relation between Fermi wave vector and density, $k_{F}=\sqrt{4 \pi n}$. We also define $\gamma=k_{F} r_{0}$ to indicate the dimensionless coupling strength of dipolar particles.

\section{Collective Modes, Zero-Point Energy, and Drag Effect}

We now consider a spin-polarized system of fermionic dipoles in a bilayer configuration. We assume that the layer densities are equal and the local hydrodynamics of the superfluid at $T=0$ to be the same as that of the normal fluid. Thus, the density response function for a single layer is approximated by [14]

$$
\chi_{0}(q, \omega) \approx \frac{m}{2 \pi} \frac{v_{F}^{2} q^{2} / 2}{\omega^{2}-v_{F}^{2} q^{2} / 2}
$$

where $v_{F}$ is the Fermi velocity. We have the Fermi velocity $v_{F}=k_{F} / m$ where $k_{F}=\sqrt{4 \pi n}$ is the Fermi wave vector. The collective modes of the bilayer system in the presence of background velocities $v_{1}$ and $v_{2}$ (in the first and second layers, respectively) are obtained from the solution of $[14,15,17]$

$$
\begin{aligned}
& \operatorname{det}\left|\begin{array}{cc}
\left(\omega-v_{1} q\right)^{2}-\frac{v_{F}^{2} q^{2}}{2}-V_{11}(q)\left(\frac{m}{2 \pi}\right) \frac{v_{F}^{2} q^{2}}{2} & -V_{12}(q)\left(\frac{m}{2 \pi}\right) \frac{v_{F}^{2} q^{2}}{2} \\
-V_{12}(q)\left(\frac{m}{2 \pi}\right) \frac{v_{F}^{2} q^{2}}{2} & \left(\omega-v_{2} q\right)^{2}-\frac{v_{F}^{2} q^{2}}{2}-V_{11}(q)\left(\frac{m}{2 \pi}\right) \frac{v_{F}^{2} q^{2}}{2}
\end{array}\right| \\
& =0
\end{aligned}
$$

From the solution of the determinant equation above we find the collective modes in the absence of any background flow $\left(v_{1}=v_{2}=0\right)$. One of them corresponds to 
the in-phase motion of dipoles in one layer with respect to the other, with the long wavelength dispersion relation

$$
\omega_{+} / E_{F} \approx \sqrt{2} \sqrt{1+\gamma}\left(q / k_{F}\right)+\frac{1-2 \tilde{a} \gamma}{2 \sqrt{2} \sqrt{1+\gamma}}\left(q / k_{F}\right)^{3}+\cdots
$$

Here $\tilde{a}=a k_{F}$ is the separation distance between the layers. The other mode corresponds to the out-of-phase motion of dipoles in one layer with respect to the other and has the dispersion relation

$$
\omega_{-} / E_{F} \approx \sqrt{2} \sqrt{1+\gamma}\left(q / k_{F}\right)-\frac{\sqrt{2} \gamma}{\sqrt{1+\gamma}}\left(q / k_{F}\right)^{2}+\cdots
$$

This second mode is expected to be more important because of its lower excitation energy. We find here that the collective modes $\omega_{+}$and $\omega_{-}$have the same leading order $q$-dependence (i.e. $\sim q$ ) and they are distinguished only at the higher order corrections. This is in contrast with other bilayer systems [14, 15, 17, 18].

For the case, $v_{1}=v_{2}=v$ which means there is no counterflow, a term $v q$ should be added to the right hand side of the above dispersion relations.

In the more general case of $v_{1} \neq v_{2}$, we may decompose the background flow into the center-of-mass flow with velocity $V=\left(v_{1}+v_{2}\right) / 2$ and counterflow with velocity $v=\left(v_{1}-v_{2}\right) / 2$. Since the center-of-mass motion plays the role of Galilean transformation we ignore it for simplicity (i.e. $V=0$ ) and concentrate on the counterflow.

The determinant equation in the presence of backflow, now yields

$$
\begin{aligned}
\omega_{+} / E_{F} \approx & \sqrt{2} \sqrt{1+\gamma}\left(q / k_{F}\right)+\frac{1-2 \tilde{a} \gamma}{2 \sqrt{2} \sqrt{1+\gamma}}\left(q / k_{F}\right)^{3}+\cdots \\
+ & {\left[\frac{\sqrt{1+\gamma}}{\sqrt{2} \gamma}+\frac{2 d(1+1 / \gamma)-1}{2 \sqrt{2} \sqrt{1+\gamma}}\left(q / k_{F}\right)+\cdots\right] \tilde{v}^{2}+\cdots }
\end{aligned}
$$

and

$$
\begin{aligned}
\omega_{-} / E_{F} \approx & \sqrt{2} \sqrt{1+\gamma}\left(q / k_{F}\right)-\frac{\sqrt{2} \gamma}{\sqrt{1+\gamma}}\left(q / k_{F}\right)^{2}+\cdots \\
& -\left[\frac{\sqrt{1+\gamma}}{\sqrt{2} \gamma}+\frac{2 d(1+11 / \gamma)-1}{2 \sqrt{2} \sqrt{1+\gamma}}\left(q / k_{F}\right)+\cdots\right] \tilde{v}^{2}+\cdots
\end{aligned}
$$

in which $\tilde{v}=\left(2 m / \hbar^{2} E_{F}\right)^{1 / 2} v$. Comparing, we find that the in-phase mode $\left(\omega_{+}\right)$is hardened while the out-of-phase mode $\left(\omega_{-}\right)$is softened by the counterflow.

The zero-point energy change due to counterflow per unit area of the bilayer may be obtained from

$$
\Delta E_{\mathrm{ZP}}=\frac{\hbar}{2} \sum_{q}\left[\Delta \omega_{+}+\Delta \omega_{-}\right]
$$

where $\Delta \omega_{ \pm}=\omega_{ \pm}(q, v)-\omega_{ \pm}(q, 0)$ is the difference between the collective mode energies with and without counterflow. An estimate involving the leading order contributions is obtained by considering the more energetic branch $\omega_{-}$and integrating 
up to $q_{\max } \sim 1 / a$ to yield

$$
\Delta E_{\mathrm{ZP}} \approx-\frac{1}{8 \pi} \frac{\sqrt{1+\gamma}}{\sqrt{2} \gamma} \frac{\tilde{v}^{2}}{\tilde{a}^{2}}
$$

That $\Delta E_{\mathrm{ZP}}$ is negative means that the total energy of the system is lowered by the counterflow. Previous calculations [17] on charged systems interacting via the Coulomb potential have shown that the bilayer separation dependence of the zeropoint energy is different for electrons and charged bosons. We, thus, surmise that a similar calculation for bilayer bosons would result in a differing layer separation dependence.

To further explore the velocity dependence of the zero-point energy, we construct the free energy by adding the kinetic energies of the fermions in each layer

$$
F=\frac{1}{2} n m\left(v_{1}^{2}+v_{2}^{2}\right)-\frac{1}{32 \pi} \frac{\sqrt{1+\gamma}}{\sqrt{2} \gamma} \frac{\left(v_{1}-v_{2}\right)^{2}}{\tilde{a}^{2}}
$$

from which we find the current densities calculated from $j_{i}=\partial F / \partial v_{i}$, to be

$$
\begin{aligned}
& j_{1}=\left(n m-\frac{\sqrt{1+\gamma}}{16 \pi \sqrt{2} \gamma \tilde{a}^{2}}\right) v_{1}+\frac{\sqrt{1+\gamma}}{16 \pi \sqrt{2} \gamma \tilde{a}^{2}} v_{2} \\
& j_{2}=\frac{\sqrt{1+\gamma}}{16 \pi \sqrt{2} \gamma \tilde{a}^{2}} v_{1}+\left(n m-\frac{\sqrt{1+\gamma}}{16 \pi \sqrt{2} \gamma \tilde{a}^{2}}\right) v_{2}
\end{aligned}
$$

The above expressions demonstrate that the supercurrent/superflow in the first (second) layer depends on the superfluid velocity on the same layer as well as that of the second (first) layer. This is the supercurrent/superfluid drag effect well known in twocomponent superfluid systems [17, 18, 20, 21] and has been discussed for a variety of related systems.

\section{Concluding Remarks}

In this paper we have studied the collective modes of bilayer dipolar Fermi gases with and without the counterflow velocities. This allowed us to obtain the change in the zero-point energy $\Delta E_{\mathrm{ZP}}$ due to counterflow. We found that $\Delta E_{\mathrm{ZP}}$ goes as $1 / a^{2}$ for dipolar fermions, presumably reflecting the particle statistics. For charged systems interacting via the Coulomb potential it was determined that $\Delta E_{\mathrm{ZP}} \sim 1 / a^{7 / 2}$ for charged bosons and $\sim 1 / a^{3}$ for electrons. Thus, the drag coefficient (i.e. the coefficient in front of $v_{2}$ in the expression for $j_{1}$ ) depends on particle statistics. It may then be possible to distinguish the quantum statistical nature of the bilayer system by measuring the drag coefficient. Therefore, it would be most interesting to extend these calculations to bilayer dipolar boson systems and boson-fermion mixtures. Qualitative and quantitive results for unequal densities, and finite layer thickness effects would also be useful to explore. 
Acknowledgements This work is supported by TUBITAK (Grant no. 109T267 and 209T059) and TUBA. We acknowledge useful discussions with S.H. Abedinpour.

\section{References}

1. S. Ospelkaus, A. Pe'er, K.-K. Ni, J.J. Zirbel, B. Neyenhuis, S. Kotochigova, P.S. Julienne, J. Ye, D.S. Jin, Nat. Phys. 4, 622 (2008)

2. M.A. Baranov, Phys. Rep. 464, 71 (2008)

3. G.M. Bruun, E. Taylor, Phys. Rev. Lett. 101, 245301 (2008)

4. G.E. Astrakharchik, J. Boronat, I.L. Kurbakov, Yu.E. Lozovik, Phys. Rev. Lett. 98, 060405 (2007)

5. H.P. Büchler, E. Demler, M. Lukin, A. Micheli, N. Prokof'ev, G. Pupillo, P. Zoller, Phys. Rev. Lett. 98, 060404 (2007)

6. N. Matveeva, S. Giorgini, arXiv:1206.3904

7. Z.-K. Lu, G.V. Shlyapnikov, Phys. Rev. A 85, 023614 (2012)

8. K.-K. Ni, S. Ospelkaus, M.H.G. de Miranda, A. Pe'er, B. Neyenhuis, J.J. Zirbel, S. Kotochigova, P.S. Julienne, D.S. Jin, J. Ye, Science 322, 231 (2008)

9. C.-K. Chan, C. Wu, W.-C. Lee, S. Das Sarma, Phys. Rev. A 81, 023602 (2010)

10. Q. Li, E.H. Hwang, S. Das Sarma, Phys. Rev. B 82, 235126 (2010)

11. N.T. Zinner, G.M. Bruun, Eur. Phys. J. D 65, 133 (2011)

12. A.G. Rojo, J. Phys. Condens. Matter 11, R31 (1999)

13. R. Asgari, B. Tanatar, B. Davoudi, Phys. Rev. B 77, 115301 (2008)

14. J.-M. Duan, Phys. Rev. Lett. 70, 3991 (1993)

15. J.-M. Duan, S. Yip, Phys. Rev. Lett. 70, 3647 (1993)

16. J.-M. Duan, Europhys. Lett. 29, 489 (1995)

17. B. Tanatar, A.K. Das, Phys. Rev. B 54(13), 827 (1996)

18. S.I. Shevchenko, S.V. Terent'ev, J. Low Temp. Phys. 23, 817 (1997)

19. D.V. Fil, S.I. Shevchenko, J. Low Temp. Phys. 30, 770 (2004)

20. A.F. Andreev, E.P. Bashkin, Sov. Phys. JETP 42, 164 (1975)

21. M.A. Alpar, S.A. Langer, J.A. Sauls, Astrophys. J. 282, 533 (1984)

22. N. Giordano, J.D. Monier, Phys. Rev. B 50, 9363 (1994)

23. X. Huang, G. Bazàn, G.H. Bernstein, Phys. Rev. Lett. 74, 4051 (1995)

24. L.A. Farina, K.M. Lewis, C. Kurdak, S. Ghosh, P. Bhattacharya, Phys. Rev. B 70, 153302 (2004)

25. S. Ishino, H. Takeuchi, M. Tsubota, J. Low Temp. Phys. 162, 361 (2011)

26. N. Matveeva, A. Recati, S. Stringari, Eur. Phys. J. D 65, 219 (2011)

27. G.F. Giulliani, G. Vignale, Quantum Theory of the Electron Liquid (Cambridge University Press, Cambridge, 2005) 